\title{
'|||||||||||||||||||||||||||||||||||||||||||||||||||||||||||||||||||.
}

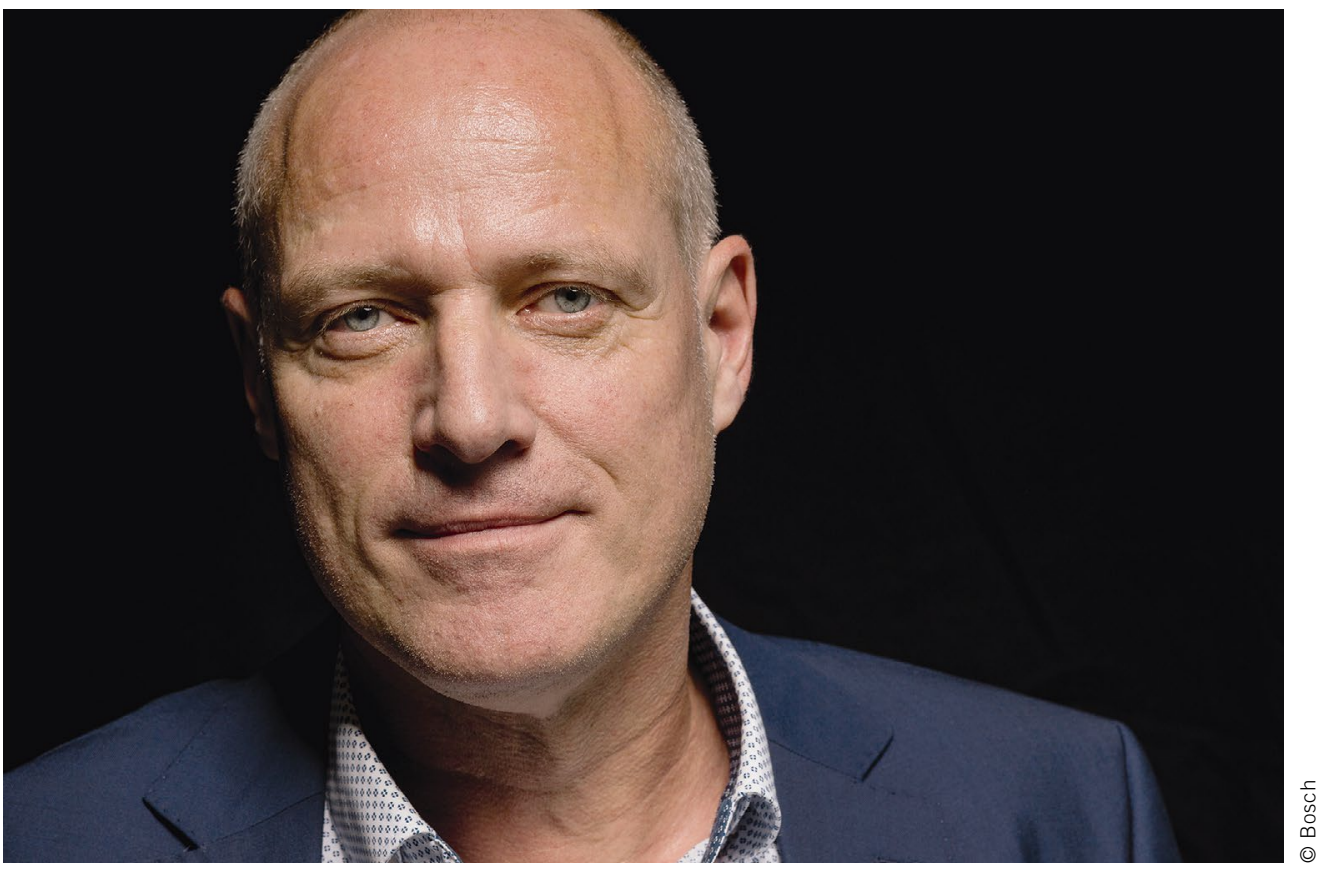

Prof. Dr.-Ing. Jürgen Hammer Senior Vice President, Business Unit Exhaust Gas Treatment Systems and Denox Components at Bosch Powertrain Solutions

\section{A Reluctance to Use Embedded AI?}

A wind of change is blowing through the offices of engine development departments that could breathe new life into a powertrain concept which some people have already written off. Or is this just hot air? Many people find it difficult to distinguish between the two things, particularly when the issue is the evaluation of embedded Artificial Intelligence (AI) functions in cars. There is no shortage of good examples of the use of AI in the automotive industry, including the image processing functions in video-based warning and braking systems that are already reliably doing their jobs in cars currently on the roads.

But why do we not hear about visions and success stories of this kind very often in connection with combustion engines or exhaust gas treatment systems? AI functions are, of course, used as development tools in these areas, but it is relatively rare for them to be embedded in engine control units. Is it because it makes no sense to use AI in combustion engines? Far from it. While it may be difficult to teach an old dog new tricks, the combustion engine, with its complex physical and chemical processes that have been constantly refined over past decades, offers significant potential for self-learning functions. Where else would it make sense to give an intelligent function the job of finding new and as yet unknown correlations between variables than in an area where the human brain has reached the often-quoted Pareto frontier?

The hardware needed to run these algorithms, which consists of high-performance ECU chips certified for automotive use, is already under development. So what are we waiting for? It is the high-quality learning material for the algorithms that is missing. "We have that already..." some developers might say, but to ensure that an AI function does not simply learn the behavior and the quirks of a few representatives of a system off by heart under test rig conditions, a huge volume of heterogeneous, clearly described data is needed. It must then be possible to extract relevant data sets from this data for the purposes of AI training. Big data analysis and data mining are familiar buzzwords in this context which describe the fundamental skills needed for AI development. Data of this kind is typically generated by car manufacturers. They have been collecting large amounts of data as part of their testing and validation activities for some time. But although we live in a networked world, it is hard for system suppliers to get their hands on this vehicle data. When they do manage to do so, the data proves to be worth its weight in gold, even if they do have to refine it themselves.

There is one possible obstacle to the development of embedded AI functions, but it can be overcome. If we succeed in putting in place agreements between manufacturers and system suppliers that allow data to be shared securely on the basis of mutual trust, we will be able to equip the combustion engine to meet future challenges by developing AI functions. That is all we need to do to achieve this. 